\title{
GHRS Observations of Massive Stars in the Large Magellanic Cloud
}

\author{
Steven N. Shore \\ GHRS Science Team/CSC \\ Code 681, Goddard Space Flight Center \\ Greenbelt, MD 20771 USA
}

Hot stars are the natural targets for ultraviolet space observations. The bulk of their energy emerges shortward of the atmospheric cutoff and nearly all of the important atomic transitions required for their analysis are located in the region shortward of $\lambda 3000 \AA$. However, since they are intrinsically rare, reddening and distance conspire to make the job of studying these important members of the stellar population of a galaxy a very difficulty task. The Goddard High Resolution Spectrograph (GHRS) adds the new dimension of photon counting to high sensitivity and resolution. This means that the massive stars can be more easily observed within our Galaxy, and also in other galaxies.

The Large Magellanic Cloud presents especially good hunting territory for the study of these stars with HST. The foreground reddening is exceptionally low. The fact that the metallicity of the Cloud is lower than the Galaxy also helps to reduce the internal reddening for stars outside of the dustiest regions. Most important is that the LMC is a site of active and comparatively recent star formation so it has a wealth of candidate massive stars for high resolution observations.

Melnick 42: Melnick 42 is one of the hottest and intrinsically brightest stars in the LMC. Classified by Walborn as an O3f star it is located to the north of the main star forming complex, the $\mathrm{R} 136 \mathrm{a}$ region. It is a relatively isolated star, although it may be surrounded by a small, faint cluster. GHRS observations, all using the G140L grating, were obtained in Nov. 1990 using both the large ( 2 arcsec) and small science $(0.25$ arcsec) apertures. Optical spectra were obtained using CASPEC at ESO. The GHRS observations have resolutions between about 800 and 2000 from $\lambda \lambda 1150-1750 \AA$, depending on the aperture. The optical/UV preliminary analysis has been discussed by Heap et al. (1991). I will summarize the results here and add a few remarks.

The most important result of the photometry is that there really are very massive stars in the world, and Mel 42 is one of them. The PC photometry (Malumuth et al. 1990) and $\mathrm{UV}$ and optical modeling of the spectrum give a mass in excess of $100 \mathrm{M}_{\odot}$. The terminal velocity is about $2900 \mathrm{~km} \mathrm{~s}^{-1}$, the mass loss rate is about $4 \times 10^{-6} \mathrm{M}_{\odot} \mathrm{yr}^{-1}, T_{\text {eff }} \approx 42500 \mathrm{~K}$, and a radius of about $28 \mathrm{R}_{\odot}$.

GHRS spectra show that the He II $\lambda 1640 \AA$ and N IV $\lambda 1718 \AA$ lines are somewhat stronger than encountered in normal O3 stars in the Galaxy and look more like the R136a spectra than a normal $\mathrm{O} 3$, as Walborn has pointed out. The N IV and N V lines are substantially stronger than a normal $\mathrm{O} 3$, or even an $\mathrm{O} 3 \mathrm{f}$, and indicate a nitrogen overabundance. The $\mathrm{N}$ abundance comes out at approximately solar, which given the metallicity of the LMC is nearly a factor of 2 overabundant. Carbon appears to be slightly depleted, to about $5 \%$ solar. Since the age of Mel 42 appears to be $\approx 2 \mathrm{Myr}$, the mixing of incomplete CNO burning products into the envelope and wind must take place quite quickly. There is nothing especially dramatic in this finding. It emphasizes, however, that the effects of nucleosynthesis are easily distinguished in stars that are not as evolved as the luminous 
blue variables or Wolf-Rayet stars.

The large aperture observations show that there are several additional early-type stars within about 1 arcsec of Mel 42. This is noted from the additional light seen in the large aperture spectrum compared with the small aperture spectrum. This is confirmed by direct examination of PC images for this region (Heap, Ebbets, and Malumuth 1991). Thus, Mel 42 seems to be similar to other very massive stars in the LMC in having formed in a small, relatively compact, cluster. The companion stars appear to be $\mathrm{B}$ type, but no details have been obtained for their spectra (although they contribute about 5 percent of the light in the G140L large aperture spectrum at $1550 \AA$ ).

An interesting observation is that the C IV profile displays analogs of the discrete absorption components (DACs) observed in Galactic stars. These are clearly seen in the C IV profile shown in Heap et al. (1991). The doublet appears as DACs at about $80 \%$ of the terminal velocity. Fits to the resonance line profiles suggest that additional turbulence, or some other fix, is required for the model. Perhaps the departures of the resonance line profiles (especially N V) from those of excited states results from anisotropic outflows? There is also the residual problem coming from the optical vs. UV analysis that the hydrogen and He II analysis gives a larger mass loss rate than indicated by fitting the CNO profiles in the UV.

Photometry of 30 Dor: Photometry by Malumuth et al. (1990) used sets of PC images to form a pseudo-color-magnitude diagram. Using the F368M and F547M filters to approximate $U$ and $V$, they find approximately 700 stars within a $5.2 \times 5.2 \operatorname{arcsec}^{2}$ region centered on R136a. They derive a luminosity function and based on Maeder (1990) derive an age of about $2 \mathrm{Myr}$ for the region and $d \ln N(M) / d \ln M \approx-1.2$ for the mass function. The luminosity function is probably complete along the main sequence down to about $\mathrm{B} 3 \mathrm{~V}$, a star that is about $9 \mathrm{M}_{\odot}$. This is not too different than other surveys have found for massive clusters. Perhaps the major difference is that this is derived from so compact a star-forming region (within about $5 \mathrm{pcs}$ ).

The R136a Spectrum: Preliminary analysis of the R136a spectrum shows that it is quite similar to that of Mel 42. The main difference is the crowded region that fills the slit at R136a. There are obviously many stars, including several WR stars and Of stars, within the 2 arcsec aperture. However, the overall profile agrees quite well with the Mel 42 convolved to the large aperture resolution. The GHRS has the ability to obtain images of the large and small apertures before or after spectroscopic observations. In the R136a case, this meant that the region could be imaged in the UV, showing the existence of at least $8 \mathrm{UV}$ bright stars (the aperture maps are performed at the nearly the same wavelength as the spectra).

I thank Sally Heap, Dennis Ebbets, Bruce Altner, Eliot Malumuth, Nolan Walborn and Ivan Hubeny for discussions. and NASA and the AAS for travel support.

Heap, S. R., Altner, B., Ebbets, D., Hubeny, I., Hutchings, J., Kudritzki, R., Voels, S., Haser, S., Pauldrach, A., Puls, J., and Butler, K. 1991, ApJL, 377, L29.

Heap, S., Ebbets, D., and Malumuth, E. 1991, in Proc. Kiel Meeting on Hot Stars (in press).

Maeder, A. 1990, $A \& A S, 84,139$.

Malumuth, E. et al. 1990, BAAS, 22, 1276. 